\title{
Fluorescence Properties of Pyrene Derivative Aggregates Formed in Polymer Matrix Depending on Concentration
}

\author{
Fuyuki Ito, ${ }^{* a, b}$ Toshifumi Kakiuchi, ${ }^{a}$ Takeshi Sakano ${ }^{a}$ and Toshihiko Nagamura ${ }^{* a}$ \\ Received (in $X X X, X X X)$ Xth $X X X X X X X X X 200 X$, Accepted Xth $X X X X X X X X X 200 X$ \\ ${ }_{5}$ First published on the web Xth $X X X X X X X X X 200 X$ \\ DOI: 10.1039/b000000x
}

We investigated the fluorescence properties of dye aggregates formed in a poly(vinylalcohol) (PVA) matrix by phase separation. Trimethyl-(2-oxo-2-pyrene-1-yl-ethyl)-ammonium bromide (PyAm) was used as a fluorescent dye molecule. The size of PyAm aggregates in the PVA thin 10 films were increased with increasing dye concentration, which was confirmed by atomic force microscope (AFM) measurements. The fluorescence spectra of PyAm in the PVA film at a lower concentration of $0.001 \mathrm{~mol} \%$ only showed the monomer emission. The fluorescence peak shifted to the red with increasing dye concentration, which was assigned to a dimer or excimer-like emission. Changes in the fluorescence spectra relate to the formation of aggregates in the films. The

15 fluorescence anisotropy decay time constant increases with increasing PyAm concentration up to the order of $100 \mathrm{ps}$. It is suggested that the exciton efficiently diffuses within the aggregates, and then was trapped at the dimer sites. We also demonstrated the application for gas sensing of nitroaromatics: 2,4-dinitrotoulene (DNT) based on the fluorescence quenching by the photoinduced electron transfer. The quenching efficiency of PyAm fluorescence reached about $43 \%$ under 20 concentration of $2.0 \mathrm{~mol} \%$. The fluorescence intensity efficiently quenched at the dimer or excimer-like band. These results indicated that the efficient fluorescence quenching increases the reaction probability between PyAm and DNT by the exciton diffusion in the aggregates, called "amplified quenching". The nano-sized aggregates of PyAm formed in the PVA films are responsible for high sensitivity as an artificial fluorescent chemosensor for vapors of the 25 nitroaromatics.

\section{Introduction}

Recently, molecular assemblies have been widely studied in photochemistry, e.g., artificial photosynthesis, photovoltaic cells, optoelectronic materials such as photoconductive 30 materials and electroluminescence devices, and so on. Molecular assemblies based on self-association or aggregation for controlled size and shape are constructed by a simple and convenient method. ${ }^{1}$ One of the features of the molecular assemblies is the development of novel functions and 35 properties, which differ from the monomer state. In the development of photo-functional materials based on organic molecules, it is important to understand the properties and functions not only for the monomer species, but also for the aggregated species.

40 Organic nanoparticles ${ }^{2}$ have been widely studied in terms of the nano science. Kasai and Nakanishi et al. first reported the optical properties of perylene nanocrystals prepared by the reprecipitation method. ${ }^{3}$ The fluorescence spectra of the nanocrystals differ from that of the bulk crystals. They

45 proposed the lattice softening in nanocrystals as a cause of the size-dependent optical properties. The size-dependent optical properties have been widely studies by several groups. ${ }^{5-16}$ Asahi and Masuhara et al. precisely studied the relation between the optical properties and crystal size by using single 50 particle fluorescence spectroscopy coupled with atomic force microscopy (AFM) ${ }^{7,8}$ It is found that the size dependence is correlated well to the surface-to-volume ratio of nanocrystals. Yao et al. reported the size dependence of emission spectra for pyrazoline nanoparticles. ${ }^{11}$ The fluorescence peaks shift to 55 red increasing the particle size. They concluded that the effects originated from the formation of charge transfer excitons, depending on the particle size. The size effect of nanoparticles comprised of conjugated polymers was also observed, which was induced by the conformational change of 60 the chain. Although the size-dependent optical properties of organic nanoparticles have been widely observed and discussed in terms of the aggregates effect, surface effect, or intermolecular interaction induced by the lattice change, the excited state dynamics of the nanocrystals have rarely been 65 studied and reported. ${ }^{14}$ Park et al. reported the enhanced emission in nanoparticles of a stilbene derivative (CNMBE). ${ }^{17}$ They also demonstrated the fluorescence ON/OFF switching due to the exposure of organic vapor in $\mathrm{CN}-\mathrm{MBE}$ nanoparticles. This is the first example for the fluorescence 70 detection of the organic vapor using organic nanoparticles. It is expected that the reaction efficiency at the surface of the nano particles/crystals will improve due to the effects of the space confinement and larger surface-to-volume ratio. However, it is considered that the efficient fluorescent 75 switching was only achieved by the suppression of nonradiative decay from the excited state (the so-called aggregated induced emission). The intermolecular interaction formed in molecular assemblies is not fully utilized in terms 
of photofunctionality. In this paper, we report our investigation of the fluorescence properties of dye aggregates formed in polymer matrix. We found a formation of nanosized aggregates of pyrene ammonium derivatives in a 5 poly(vinylalcohol) (PVA) matrix by phase separation. The photophysical properties of nano-sized pyrene derivatives are discussed as a function of the concentration in PVA films. The application of the nano-aggregates to the nitro aromatics vapor sensor is also demonstrated as a function of the dye 10 concentration.

\section{Experimental}

The pyrene ammonium derivative (trimethyl-(2-oxo-2pyren-1-yl-ethyl)-ammonium bromide; PyAm, Fig.1) was synthesized according to the previous report. ${ }^{18,19}$ The PyAm 15 doped PVA thin films were prepared by spin-coating or dropcasting onto quartz plates. The PVA concentration in ionexchanged and distilled water was fixed to $0.113 \mathrm{~mol} \cdot \mathrm{dm}^{-3}$ $(0.5 \mathrm{wt} \%)$. To change the PyAm molecular fraction in the films, concentrations of PyAm in PVA aqueous solutions were ${ }_{20} 0.01,0.05,0.1,0.5,1.0$, and $2.0 \mathrm{~mol} \%$ for monomer units. The coated films were dried under vacuum condition for $24 \mathrm{~h}$ at room temperature. UV-Vis absorption spectra were measured by a Hitachi U-4100 spectrometer. Fluorescence spectra were recorded by a Hitachi F-4500 fluorescence 25 spectrophotometer. X-ray diffraction (XRD) patterns were obtained using an X-ray diffractometer (Rigaku Co., Ltd. MultiFlex, $\mathrm{Cu} \mathrm{K} \alpha(\lambda=1.54 \AA)$ radiation) at room temperature. The morphology of the films was measured by AFM (SPA300HV, SII NanoTechnology Inc.). Fluorescence 30 lifetimes were measured by a single photon counting method using a streakscope (Hamamatsu Photonics, C4334-01). ${ }^{20,21}$ The sample solutions were excited with SHG $(\lambda=430 \mathrm{~nm})$ of a mode-locked Ti:sapphire laser (Spectra-Physics, Tsunami, fwhm: $1.5 \mathrm{ps}$ ) equipped with a pulse selector and a harmonic 35 generator. The laser light incidents from the substrate side of the sample under the attenuated total reflection condition via a prism. The fluorescence decays were measured with magicangle emission polarization to avoid affects the decay profiles. Time-resolved fluorescence anisotropy was obtained by 40 changing the detection polarization on the fluorescence path parallel or perpendicular to the polarization of the excitation light. The anisotropy decays then were calculated as follows: $r(t)=\left[I_{\mathrm{VV}}(t)-G I_{\mathrm{VH}}(t)\right] /\left[I_{\mathrm{VV}}(t)+2 G I_{\mathrm{VH}}(t)\right]$ where $I_{\mathrm{VV}}(t)$ (or $\left.I_{\mathrm{VH}}(t)\right)$ is the fluorescence decay when the excitation light is 45 vertically polarized and only the vertically (or horizontally) polarized portion of fluorescence is detected. The first and second subscripts represent excitation and detection polarization, respectively. The factor $G$ is defined by $I_{\mathrm{VV}}(t) / I_{\mathrm{VH}}(t)$, which is equal to the ratio of the sensitivities of 50 the detection system for vertically and horizontally polarized light. The polarized angle of excitation light was adjusted by using the Berek compensator (Newfocus Model 5540). All experiments were carried out at room temperature.

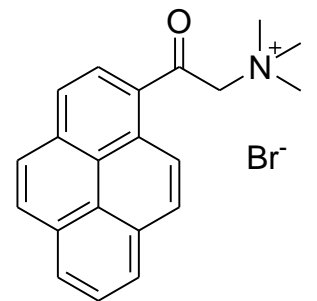

$55 \quad$ Fig. 1 Molecular structure of pyrene ammonium derivative.

\section{Results and Discussion}

The absorption spectra of PyAm in PVA films are shown in Fig.2. The absorpiton peaks observed at 370 and $410 \mathrm{~nm}$. Although the concentration dependence was not observed in 60 the spectral shape, the concentration between films 0.10 and $2.00 \mathrm{~mol} \%$ increases about 20 times but the absorbance only increase less than about 10 times. It is suggested that intermolecular interaction between PyAm most probably exist in the ground state. Fig. 3 shows the fluorescence spectra of ${ }_{65}$ PyAm in PVA films excited at $370 \mathrm{~nm}$ as a function of concentration. In the case of $0.01 \mathrm{~mol} \%$, the fluorescence peak was observed around $450 \mathrm{~nm}$. The fluorescence peaks shifted to the red with increasing concentration. The fluorescence peak reached at $530 \mathrm{~nm}$ under the concentration 70 of $2.0 \mathrm{~mol} \%$, which is identical to the fluorescence spectrum of PyAm powder. The excitation spectra of PyAm in PVA films is unchanged in all concentration. We examined the structure and morphology of the films by XRD and AFM, respectively. Fig. 4 shows the XRD curve of PyAm in PVA 75 films as a function of concentration. The black line in Fig.4 indicates the diffraction pattern of PyAm power. In the concentration of $2.0 \mathrm{~mol} \%$, the diffraction peak was observed at $2 \theta=11.1 \mathrm{deg}(d=0.80 \mathrm{~nm})$, which indicates the formation of PyAm crystals in the PVA matrix. All other diffraction 80 peaks were either very weak or undetectable. No diffraction peak was observed for concentrations less than $1.0 \mathrm{~mol} \%$. The AFM images of PyAm in PVA films are shown in Fig. 5 for concentrations of $0,0.1$ and $2.0 \mathrm{~mol} \%$. The film thicknesses evaluated by ellipsometry was approximately $13.8 \pm 1.2 \mathrm{~nm}$. ${ }_{85}$ The root-mean-square roughness of PVA film is $0.16 \mathrm{~nm}$ in the absence of PyAm, which is the relatively flat surface. The domain with height of few nm was observed in the presence of PyAm. The domain width is increasing with increasing of PyAm concentration as shown in Fig.5. The nano-sized 90 domains with about 100 to $200 \mathrm{~nm}$ width were observed at 2.0 mol\%. The crystallization is caused by increasing of the local concentration during the solvent evaporation. These findings suggest PyAm formed nano-crystals depending on the concentration in the PVA films most probably by the phase 95 separation. 


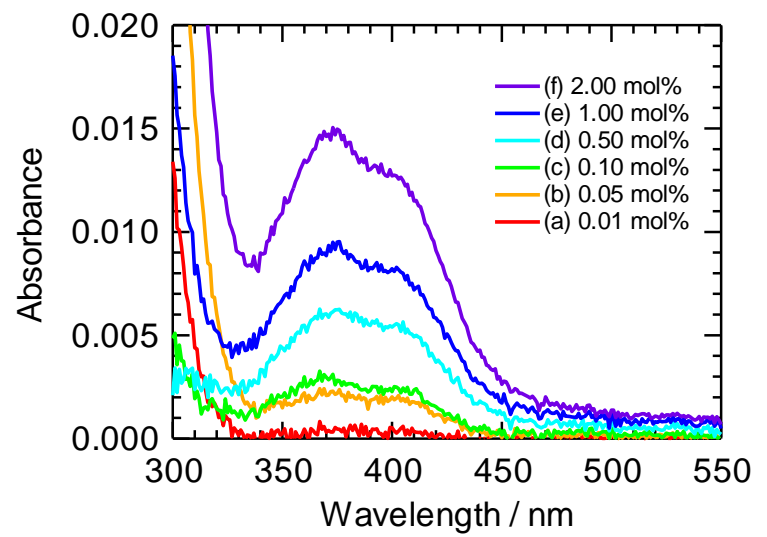

Fig. 2 Absorption spectra of $\mathrm{f}$ PyAm in PVA films as a function of concentration.

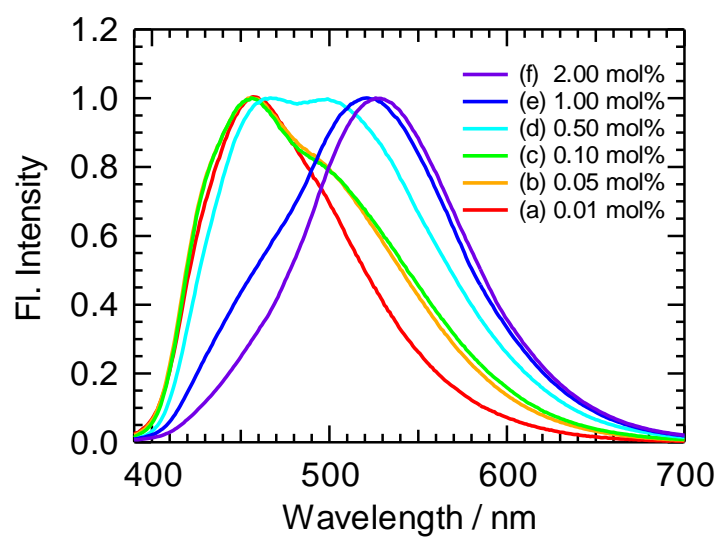

Fig. 3 Fluorescence spectra of PyAm in PVA films excited at $370 \mathrm{~nm}$ as a function of concentration.

To discuss the fluorescence spectral change, the fluorescence spectra were analyzed as a function of 10 concentration, which are shown in Fig.6. We assumed the fluorescence spectra of PyAm in PVA films are consisted of three species: a monomer, a partially overlapped dimer (dimer-1) and a fully overlapped dimer (aggregated species, dimer-2). The emission spectrum at $0.001 \mathrm{~mol} \%$ (much less 15 concentration) was used as the monomer fluorescence. The spectrum of the fully overlapped dimer was fitted by a Gaussian function of fluorescence from PyAm powder. The spectrum of partially overlapped dimer was assumed by difference spectrum between the monomer and the dimer-2 20 from the observed spectra. The observed spectra at each concentration were reproduced by the summation of the three species with non-linear least square fitting. Fig.7 shows fractions of three components estimated from observed spectra of PyAm in PVA films as a function of concentration.
25 The monomer fraction monotonically decreased with increasing concentration. The dimer-1 fraction was slightly increased with concentrations below $0.5 \mathrm{~mol} \%$, and decreased considerably after that. The dimer- 2 was not observed at concentrations less than $0.1 \mathrm{~mol} \%$. The fraction was gradually 30 increased above $0.5 \mathrm{~mol} \%$ in concomittant decrease of the dimer-1. The fully overlapped species corresponds to the fluorescence from PyAm crystals. The AFM observation suggests that the domain size depends on the PyAm concentration. Therefore, the nano-crystals and/or aggregates 35 formed by phase separation are presumed to show hierarchal change (like a consecutive reaction) of fluorescence property depending on the domain size.

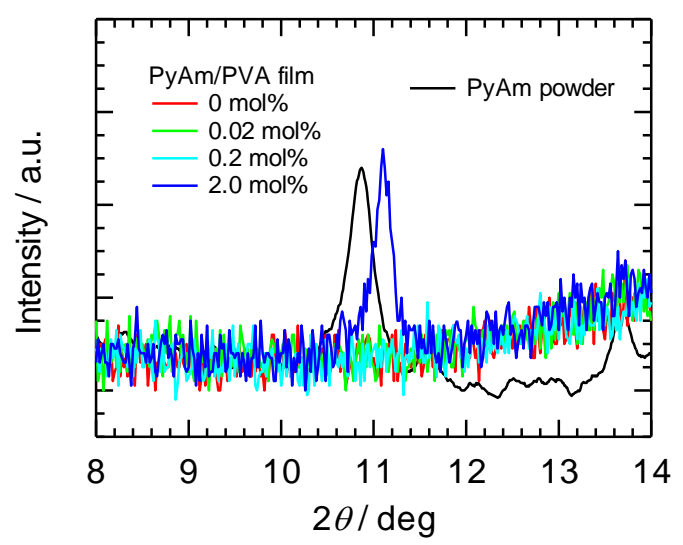

Fig. 4 XRD curves of PyAm in PVA and solid powder (black line).
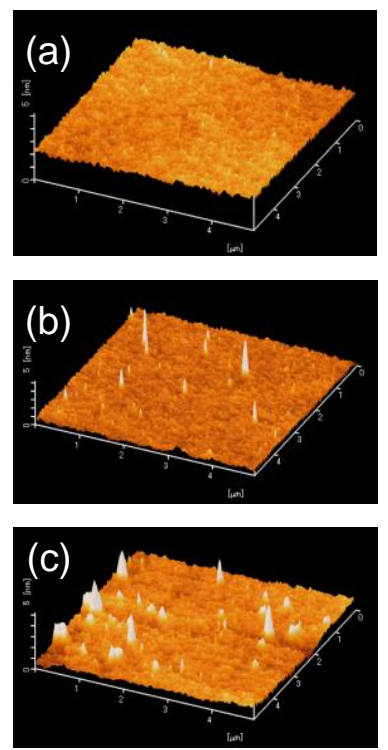

Fig. 5 AFM image of PyAm in PVA films. The concentration of PyAm was (a) 0 (b) 0.1 (c) $2.0 \mathrm{~mol} \%$. 

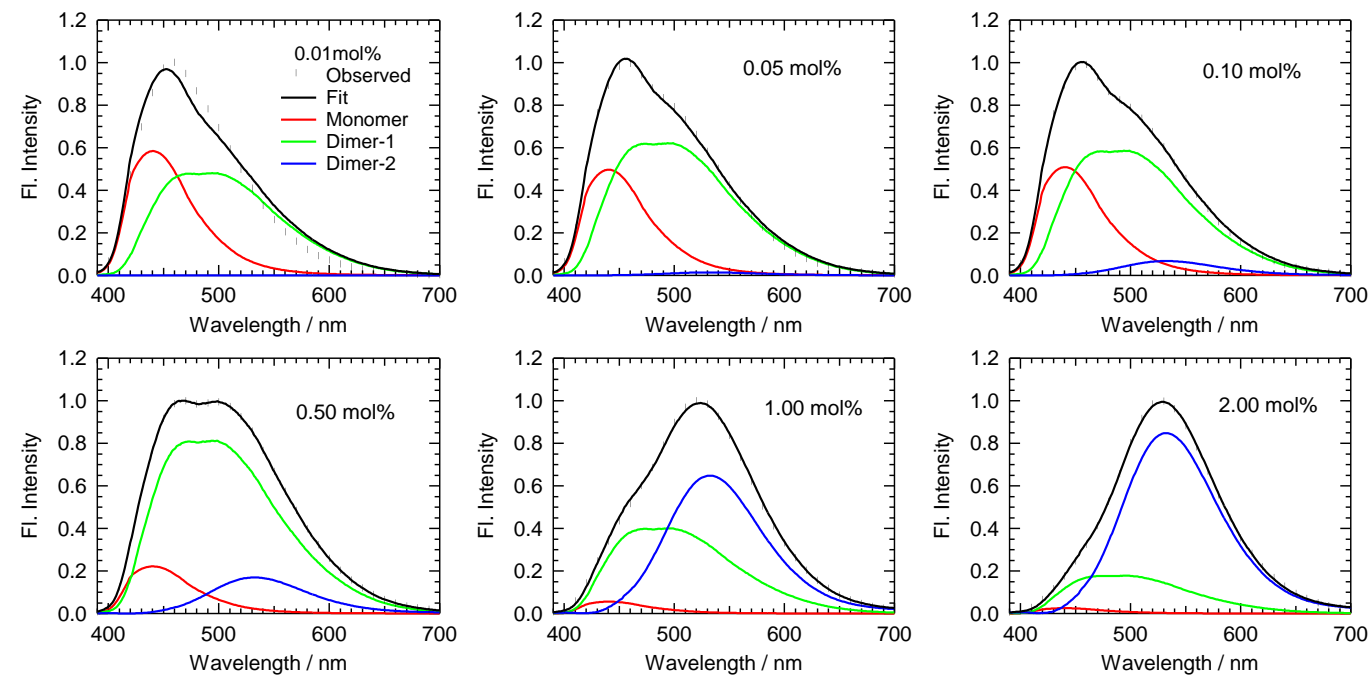

Fig. 6 Fluorescence spectra of PyAm/PVA films as a function of dye concentration excited at $370 \mathrm{~nm}$. Closed circles and solid line depict the observed fluorescence spectra and simulated spectra from summation of relative abundance three types of fluorescence species by using least square fitting, respectively.

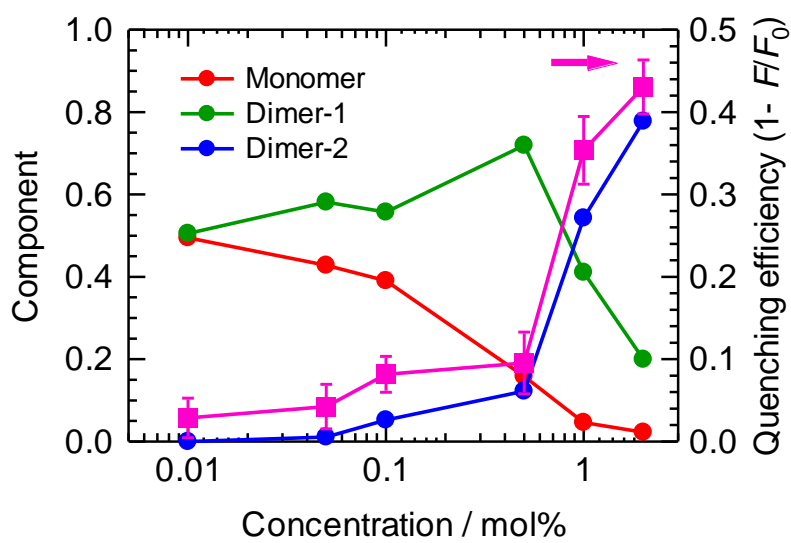

Fig. 7 Relative components of emissive species of PyAm in PVA as a function of concentration (left hand axis). The fluorescence quenching efficiency of PyAm just after immersing DNT vapor is also indicated as the pink line.

Next, we demonstrated the application of PyAm nanoaggregates fluorescence to nitroaromatics vapor. The fluorescence is quenched by the photoinduced electron 15 transfer from excited PyAm to dinitrotoluene (DNT). The free energy change $(\Delta G)$ of the electron transfer quenching was approximately estimated to be $-0.78 \mathrm{eV}$ with oxidation potential $(1.02 \mathrm{~V}$ (vs. $\mathrm{Ag} / \mathrm{AgCl})$ in aqueous solution), and excitation energy $(2.76 \mathrm{eV})$ of PyAm and reduction potential 20 of DNT $(-0.96 \mathrm{~V}$ (vs. $\mathrm{Ag} / \mathrm{AgCl})$ ) by the Rehm-Weller equation. ${ }^{22}$ The Coulomb term was neglected in this estimation. The quenching ability was tested upon exposing the films to saturated vapors of DNT for $10 \mathrm{~min}$. The fluorescence intensity of PyAm doped in PVA film at 0.01 $25 \mathrm{~mol} \%$ was not changed before and after immersion. On the other hand, the fluorescence intensity of PyAm at $2.0 \mathrm{~mol} \%$ in PVA films was dramatically quenched by about $43 \%$. This fluorescence quenching originated from the photoinduced electron transfer reaction from excited PyAm to DNT. We 30 measured the concentration dependence of the fluorescence quenching efficiency as shown in Fig.7. The quenching efficiency slightly increased up to about $0.5 \mathrm{~mol} \%$, and then the efficiency dramatically increased. This behavior is identical to the fraction of dimer- 2 species obtained by the 35 analysis of the fluorescence spectra. It is thus strongly suggested that the quenching efficiency is related to the amount of the dimer-2 species. The intermolecular interaction in the PyAm aggregates formed by the phase separation in PVA films is a significant factor for the efficient fluorescence 40 quenching.

Swager has proposed the molecular wire method based on the $\pi$-conjugated polymer to detect vapors of nitroaromatics at ppm order. ${ }^{23,24}$ The $\pi$-conjugated polymer has the ability to effectively transport the exciton along the polymer chain to 45 result in amplified quenching. The amplified quenching occurs in organized media such as a Langumuir-Blogett (LB) monolayer. We have observed amplified quenching of carbazolyl derivatives in the LB monolayer films by a trace amount of acceptor (quencher). ${ }^{25-27}$ For example the ${ }_{50}$ squarylium dye $\mathrm{LB}$ films can detect $\mathrm{NO}_{2}$ gas at the parts-perbillion level. ${ }^{28,29}$ In our device combined with SPR excitation and DNA matrix, the $\mathrm{NO}_{2}$ gas can be detected at $0.1 \mathrm{ppm}$ level. ${ }^{30}$ In the present case, it can be presumed that the exciton diffuses in the nano-crystals or aggregates, which will 55 contribute to increments of the apparent reaction radius. To directly clarify the exciton dynamics, a fluorescence depolarization method was applied. ${ }^{20}$ The anisotropy decay curves show a direct relationship with the diffusion process. Fig.8 shows fluorescence anisotropy decay of PyAm in PVA ${ }_{60}$ films as a function of concentration. Fluorescence anisotropy decay was reproduced as a double exponential function with a constant value $\left(r_{\infty}\right)$. The time constants of the decay were summarized in Table 1. The rate constant of the anisotropy decay was increased with increasing the PyAm concentration. 
This indicates that the excitation energy migration efficiently occurred in the PyAm crystal as a function of concentration. The energy migration in nano-aggregates will be related to their domain size, which was confirmed by the AFM 5 observation (Fig.5). The $r_{\infty}$ value is decreased with increasing the concentration, suggesting the exciton diffusion occurred efficiently within larger nano-aggregates. The fluorescence decay analysis also strongly suggested that the excitation energy migration efficiently occurred in the PyAm crystal 10 depending on the concentration (Electronic Supplementary Information). ${ }^{20}$ The nanocrystals or aggregates have the advantage of not only efficient exciton diffusion; but also a larger surface-to-volume ratio preferable to gas sensing or adsorption.

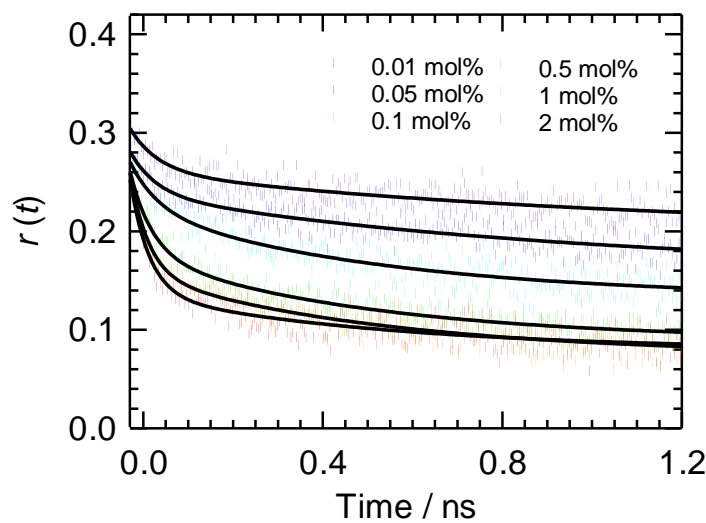

15

Fig. 8 The fluorescence anisotropy decay of PyAm in PVA films as a function of concentration excited at $\lambda=430 \mathrm{~nm}$ laser pulse monitored around $\lambda=440-470 \mathrm{~nm}$.

Table 1. Fluorescence anisotropy decay of PyAm in PVA films as a 20 function of concentration.

\begin{tabular}{cccccc}
\hline $\mathrm{mol} \%$ & $r_{\infty}$ & $\mathrm{A}_{1}$ & $\tau_{1} / \mathrm{ps}$ & $\mathrm{A}_{2}$ & $\tau_{2} / \mathrm{ns}$ \\
\hline 0.01 & 0.20 & 0.027 & 63 & 0.059 & 1.07 \\
0.05 & 0.16 & 0.026 & 58 & 0.076 & 0.98 \\
0.10 & 0.13 & 0.029 & 62 & 0.086 & 0.56 \\
0.50 & 0.09 & 0.046 & 50 & 0.083 & 0.52 \\
1.00 & 0.07 & 0.049 & 41 & 0.079 & 0.55 \\
2.00 & 0.07 & 0.061 & 45 & 0.055 & 0.67 \\
\hline
\end{tabular}

\section{Conclusions}

We have found the fluorescence spectral change of pyrene derivatives in nano-crystals or aggregates formed by phase 25 separation in polymer matrices depending on the domain size. We also demonstrated its application to gas sensing of nitroaromatics based on the fluorescence quenching by photoinduced electron transfer. The efficient fluorescence quenching was due to increased reaction probability between 30 PyAm and nitroaromatics by the exciton diffusion in the aggregates. The sensing ability will be improved by optimizing the molecular design, crystal size, and/or polymer matrix. Detailed studies are now in progress to determine the size-dependent fluorescence properties and effects of using a 35 substitution group for PyAm coupled with dynamics of crystal nucleus formation.

\section{Acknoledgements}

The authors express sincere gratitude to Prof. Keiji Tanaka for AFM measurements. This work was partly supported by ${ }_{40}$ Grand-in-Aids for Research in Priority Area (No. 470: Strong Photon-Molecule Coupling Fields, T.N.), Grant-in-Aid for Young Scientist (B) (No. 21750021, F.I.) from the Ministry of Education, Culture, Sports, Science, and Technology (MEXT) of the Japanese Government. F.I. was also suported by 45 Inamori Foundation and Iketani Science and Technology Foundation.

\section{Notes and references}

${ }^{a}$ Department of Applied Chemistiry, Faculty of Engineering, Kyushu University,744 Motooka, Nishu-ku, Fukuoka 819-0395, Japan, Fax: +8150 92-802-2880; Tel: +81-92-802-2880; E-mail:nagamura@cstf.kyushuu.ac.jp

${ }^{b}$ Present Address: Department of Chemistry, Faculty of Education, Shinshu University, 6-ro, Nishinagano, Nagano 380-8455, Japan. Fax: +81-26-238-4114; Tel: +81-26-238-4114; E-mail: fito@shinshu-u.ac.jp

$55 \dagger$ Electronic Supplementary Information (ESI) available: Fluorescence decay profiles of PyAm in PVA films. See DOI: 10.1039/b000000x/

1 G. M. Whitesides, B. Grzybowski, Science 2002, 295, 2418-2421.

2 H. Masuhara, H. Nakanishi, K. Sasaki, Eds., Single Organic Nanoparticles; Springer: Berlin, 2003.

603 H. Kasai, H. S. Nalwa, H. Oikawa, S. Okada, H. Matsuda, N. Minami, A. Kakuda, K. Ono, A. Mukoh, H. Nakanishi, Jpn. J. Appl. Phys. 1992, 31, L1132.

4 H. Kasai, H. Kamatani, Y. Yoshikawa, S. Okada, H. Oikawa, A. Watanabe, O. Itoh, H. Nakanishi, Chem. Lett. 1997, 1181-1182.

655 H. Oikawa, T. Mitsui, T. Onodera, H. Kasai, H. Nakanishi, T. Sekiguchi, Jpn. J. Appl. Phys. Part 2 - Lett. 2003, 42, L111-L113.

6 N. Kurokawa, H. Yoshikawa, N. Hirota, K. Hyodo, H. Masuhara, ChemPhysChem 2004, 5, 1609-1615.

7 T. Asahi, H. Matsune, K. Yamashita, H. Masuhara, H. Kasai, H. 70 Nakanishi, Pol. J. Chem. 2008, 82, 687-699.

8 T. Asahi, T. Sugiyama, H. Masuhara, Acc. Chem. Res. 2008, 41, 1790-1798.

9 T. Tachikawa, H. R. Chung, A. Masuhara, H. Kasai, H. Oikawa, H. Nakanishi, M. Fujitsuka, T. Majima, J. Am. Chem. Soc. 2006, 128, 15944-15945.

10 H. B. Fu, J. N. Yao, J. Am. Chem. Soc. 2001, 123, 1434-1439.

11 D. B. Xiao, X. Lu, W. S. Yang, H. B. Fu, Z. G. Shuai, Y. Fang, J. N. Yao, J. Am. Chem. Soc. 2003, 125, 6740-6745.

12 S. Y. Li, L. M. He, F. Xiong, Y. Li, G. Q. Yang, J. Phys. Chem. B 2004, 108, 10887-10892.

13 J. K. Grey, D. Y. Kim, B. C. Norris, W. L. Miller, P. F. Barbara, J. Phys. Chem. B 2006, 110, 25568-25572.

14 Bhongale, C. J.; Chang, C. W.; Lee, C. S.; Diau, E. W. G.; Hsu, C. S. J. Phys. Chem. B 2005, 109, 13472-13482.

8515 A. Patra, N. Hebalkar, B. Sreedhar, M. Sarkar, A. Samanta, T. R. Radhakrishnan, Small 2006, 2, 650-659.

16 T. Seko, K. Ogura, Y. Kawakami, H. Sugino, H. Toyotama, J. Tanaka, Chem. Phys. Lett. 1998, 291, 438-444.

17 An, B. K.; Kwon, S. K.; Jung, S. D.; Park, S. Y. J. Am. Chem. Soc. 2002, 124, 14410-14415.

18 N. Nakashima, Y. Tomonari, H. Murakami, Chem. Lett. 2002, 638639.

19 N. Nakashima, Y. Tanaka, Y. Tomonari, H. Murakami, H. Kataura, T. Sakaue, K. Yoshikawa, J. Phys. Chem. B 2005, 109, 1307613082.

20 F. Ito, T. Kakiuchi, T. Nagamura, J. Phys. Chem. C 2007, 111, $6983-$ 6988.

21 T. Kakiuchi, F. Ito, T. Nagamura, J. Phys. Chem. B 2008, 112, 39313937. 
22 D. Rehm, A. Weller, Isr. J. Chem. 1970, 8, 259-271.

23 J. S. Yang, T. M. Swager, J. Am. Chem. Soc. 1998, 120, $11864-$ 11873.

24 D. T. McQuade, A. E. Pullen, T. M. Swager, Chem. Rev. 2000, 100, 5 2537-2574.

25 T. Nagamura, S. Kamata, T. Ogawa, Nippon Kagaku Kaishi 1987, 2090-2094.

26 T. Nagamura, S. Kamata, K. Toyozawa, T. Ogawa, Ber. Bunsenges. Phys. Chem. 1990, 94, 87-91.

1027 T. Nagamura, K. Toyozawa, S. Kamata, Colloids and Surfaces A 1995, 102, 31-37.

28 M. Furuki, K. Ageishi, S. Kim, I. Ando, L. S. Pu, Thin Solid Films 1989, 180, 193-198.

29 M. Furuki, L. S. Pu, Thin Solid Films 1992, 210/211, 471-473.

1530 T. Nagamura, M. Yamamoto, M. Terasawa, K. Shiratori, Appl. Phys. Lett. 2003, 83, 803-805. 